\title{
E17 NAPL RECOVERY FROM LOW PERMEABLE LAYERS
}

\author{
A.H. DE ZWART, J. BRUINING, AND A. DE VRIES
}

\begin{abstract}
A one-dimensional model that describes fluid flow from low permeable reservoir parts exposed on one end to steam temperature has been formulated. There is a no flow condition at the other end. Heat penetrates into the low permeable reservoir by conduction. The temperature profile is obtained from an experiment designed to quantify product recovery from indirectly heated reservoir parts. Preliminary results show a poor recovery from non-volatile components.
\end{abstract}

\section{INTRODUCTION}

Steam injection is a promising method for the remediation of oil spills. An effective well configuration consists of placing steam injection wells within and around the contaminated soil, and extraction wells within the contaminated region. The oily contaminants are produced in the extraction wells together with condensate water and air from the extraction well. Around the injection well an expanding heated zone of steam is formed. The steam directly heats permeable parts. Less permeable parts are heated indirectly by conduction. In the directly heated parts steam displaces the fluids towards the production well and enhances desorption of the contaminants from the solid surfaces. Volatile components will boil off both from the directly and indirectly heated regions. The most important advantage of steam lies in the fact that the vapor pressures of the water and oily contaminants are approximately additive. Therefore compounds with a high boiling point also evaporate and move along with the steam. The additional advantage is that the vapor pressure in indirectly heated contaminated parts is higher than in the already swept parts leading to a driving force from within the contaminated regions. In this paper we want to show the recovery from indirectly heated parts in the subsurface with a 1-D steam model. Our aim is to investigate whether non-volatile components are also partly recovered.

For a literature overview we refer to [11], [7]. Most of the studies deal with product or oil recovery due to steam drive [13]. There is a lack of interest in the recovery from indirectly heated reservoir parts. It can be expected that the indirect product recovery is one of the major advantages of the application of steam for remediation of sites polluted by oily contaminants for the reasons explained above. Indeed thermal diffusion is about ten thousand times as fast as mass diffusion leading to hundred times faster clean up times.

Therefore we address the problem that heat penetrates by conduction in a low permeable 1-D segment, that is connected to a free out flow part on one side and closed with a no flow condition on the other. The problem is of practical relevance for the quantification of the recovery of an oily contaminant consisting of a mixture of dead oil and volatile oil trapped in a low permeable lens. The problem is studied both experimentally and theoretically. Experimentally we study the flow from a tube filled with sand, which is closed at one end

Key words and phrases. Porous medium, remediation with steam, Indirect heating, Distillation. a.h.dezwart@citg.tudelft.nl, J.Bruining@ta.tudelft.nl, A.S.DEVRIES@siep.shell.com. 


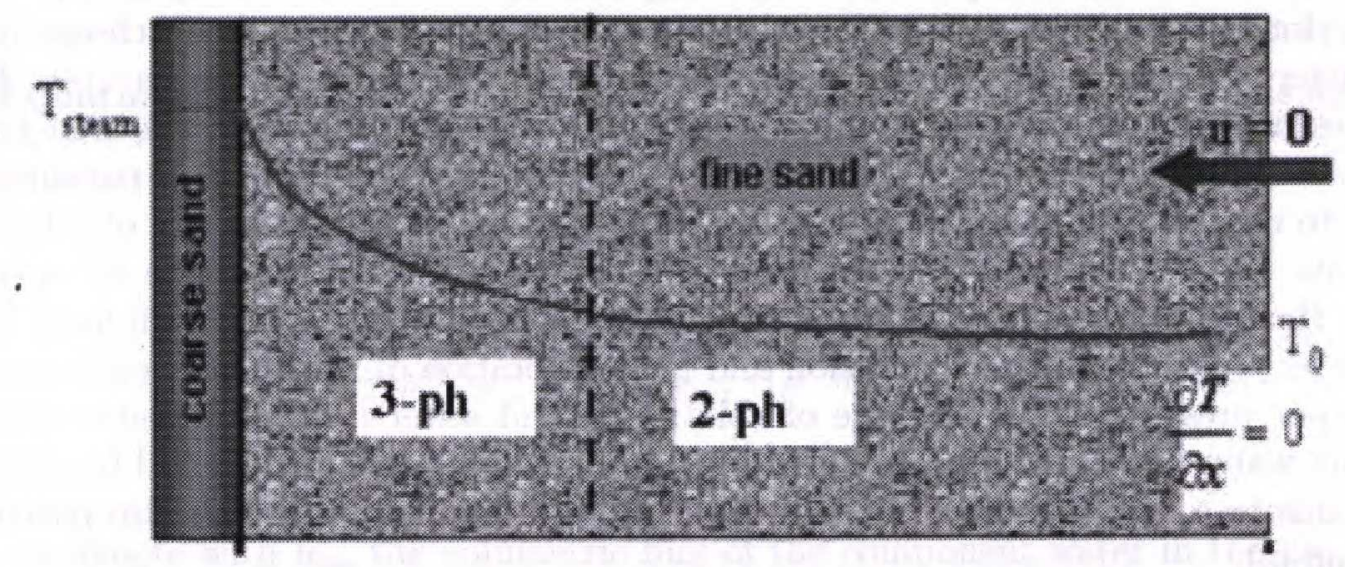

Figure 3.1. An isolated sand body with an open end exposed to steam

and heated by a heating element at the other side, which also is the production end. We use the measured temperature profiles, which are input in a theoretical flow model that assumes local thermodynamic equilibrium. The main mechanisms for product recovery are thermal expansion and evaporation.

\section{Notation AND SYMBOLS}

Throughout the text we indicate the components and phases as superscripts. The first index $a, o, g$ denotes the phase i.e. aqueous, oleic and gaseous. The second index $w, d, v$ denotes the components i.e. water, dead oil and volatile oil. Capital letters indicate pure components and small letters denote the components in a mixture. For instance $\rho^{o v}$ denotes the concentration of volatile oil in the oleic phase whereas $\rho^{o V}$ denotes the density of pure volatile oil. Other symbols are defined in the Table in the Appendix.

\section{PhysicAl MODEL}

We consider the 1-D flow of vapor, water, volatile oil and dead oil in a low permeable lens of length $L$ towards a steam-flooded higher permeable medium (see Figure 3.1). The far upstream side of the lens satisfies a no flow condition. The lens is filled with water at saturation $S^{w i}$ and oil. This geometry is representative for a layered system with high permeability contrast, where the flow in the low permeable layer is almost perpendicular to the layer direction.

Characteristic of the dead oil is that it has a zero vapor pressure at all temperatures of interest. The volatile oil consists of a single component with a boiling point $T^{v}\left(p^{a v}\right)$ above the initial temperature and a vapor pressure of the same order of magnitude as steam at the boiling point of water. $p^{a v}$ is the prevailing pressure. At high temperatures there are three phases i.e. including a gas-phase and at lower temperatures there is only the aqueous and the oleic phase. All components are considered to be in local thermodynamic equilibrium. 
As our interest is in interpretation of the experiment we consider a simplified model of heat conduction in the porous medium and the iron tube that leads with a freely chosen interaction parameter to a sufficiently accurate representation of the measured temperatures. The model assumes heat conduction in the axial direction both in the sand and the iron tube and a heat transfer term between the two. The heat transfer coefficient is a free parameter. A full model to describe the performance in the field requires the incorporation of a heat balance equation. This is a straightforward extension, which is, however, outside the scope of this paper. Heat penetrates from one side into the tube. Flow towards the high perm medium is caused by (1) thermal fluid expansion and (2) evaporation of the volatile oil-water mixture. The vapor pressure of the mixture of volatile oil and water in the low perm layer exceeds the pure water vapor pressure in the high perm medium, which is depleted from volatile oil. This leads to a net driving force towards the high perm medium, which also partly removes the dead oil.

We will assume a constant pressure and therefore the excess volume is produced instantaneously i.e. the fluids at constant composition and temperature are incompressible. Such an approximation is warranted if the characteristic time $\varphi \mu c_{t} L^{2} / k$ at which pressure relaxation occurs is much smaller than the characteristic time $(\rho c)_{e f f} L^{2} / \lambda$ for the heating rate. $c_{t}$ is the effective compressibility. In other words all fluids inclusive the gases remain at constant pressure, and have a volume that only depends on the temperature and composition.

The mixture of dead oil and volatile oil behave as an ideal fluid with zero heat of mixing and there are no volume effects upon mixing. There are also no volume effects in the gaseous phase. Therefore we can write

$$
\frac{\rho^{o v}}{\rho^{o V}}+\frac{\rho^{o d}}{\rho^{o D}}=1, \quad \frac{\rho^{g v}}{\rho^{g V}}+\frac{\rho^{g w}}{\rho^{g W}}=1
$$

The ratios of the densities e.g. $\frac{\rho^{o v}}{\rho^{o V}}$ can be interpreted as the volume fraction of the component of interest in the phase and will be denoted by $\varsigma^{o v}, \varsigma^{o d}, \varsigma^{g v}, \varsigma^{g w}$ respectively. Moreover we assume that the volatile oil and the water in the gaseous phase behave as ideal gases. The phase behavior is explained in the section below. All other properties are summarized in the appendix.

As to the thermodynamic quantities we disregard any small pressure deviation that arises due to the flow of fluids i.e. we assume that all thermodynamic quantities only depend on the constant average pressure $p^{a v}$.

Now suppose we have a mole fraction $x_{A}$ of volatile oil in the liquid oil phase. Then we use Raoult's law and thus is the vapor pressure of the oil phase given by the vapor pressure of the pure heptane times the mole fraction. Of this mixture we can determine the "azeotropic temperature" $T_{z}$ with the equation

$$
\begin{gathered}
P^{t o t}=x^{o v} P^{b} \exp \left(\frac{-\Lambda^{V}\left(T^{b V}\right) M^{V}}{R}\left(\frac{1}{T_{z}}-\frac{1}{T^{b V}}\right)\right)+ \\
+P^{b} \exp \left(\frac{-\Lambda^{W}\left(T^{b W}\right) M^{W}}{R}\left(\frac{1}{T_{z}}-\frac{1}{T^{b W}}\right)\right):=P^{g v}+P^{g w}
\end{gathered}
$$

where the parameters are defined in the Table of the appendix. This defines the volatile oil mole fraction temperature relation $x^{o v}-T$ in the three phase hot zone. 


\section{MOdel Equations}

4.1. Equations in the hot three phase zone. The mass balance equations for the three components in the three phase zone can be written as

$$
\begin{gathered}
\varphi \frac{\partial}{\partial t}\left(\rho^{W} S_{w}+\rho^{g w} S_{g}\right)+\frac{\partial}{\partial x}\left(\rho^{W} u_{w}+\rho^{g w} u_{g w}\right)=0 \\
\varphi \frac{\partial}{\partial t}\left(\rho^{o v} S_{o}+\rho^{g v} S_{g}\right)+\frac{\partial}{\partial x}\left(\rho^{o v} u_{o v}+\rho^{g v} u_{g v}\right)=0 \\
\varphi \frac{\partial}{\partial t}\left(\rho^{o d} S_{o}\right)+\frac{\partial}{\partial x}\left(\rho^{o d} u_{o d}\right)=0
\end{gathered}
$$

Here we denote with $u_{g w}$ the volumetric flux of the component water in the gas phase etc. This velocity is not equal to the the velocity of volatile oil in the oil phase $u_{g v}$ due to diffusion. We disregard dispersion effects, which is warranted in the gas phase. However, dispersion/diffusion in the liquid oil phase is small. A lengthy derivation, (see [5] for a full derivation), leads to

$$
\frac{\rho^{g w}}{\rho^{g W}}\left(u_{g w}-u_{g}\right)=-\mathcal{D}^{w v} \frac{\partial \zeta^{g w}}{\partial x}, \quad \frac{\rho^{g v}}{\rho^{g V}}\left(u_{g v}-u_{g}\right)=-\mathcal{D}^{w v} \frac{\partial \zeta^{g v}}{\partial x}
$$

and

$$
\frac{\rho^{o v}}{\rho^{o V}}\left(u_{o v}-u_{o}\right)=-\mathcal{D}^{d v} \frac{\partial \zeta^{o v}}{\partial x}, \quad \frac{\rho^{o d}}{\rho^{o D}}\left(u_{o d}-u_{o}\right)=-\mathcal{D}^{d v} \frac{\partial \zeta^{o d}}{\partial x}
$$

where $u_{g}$ and $u_{o}$ are volume averaged phase velocities (see [2]) and not mass averaged velocities. In deriving 4.4 and 4.5, we assumed that the thermal expansivities of water vapor and volatile oil vapor and also the expansivities of volatile and dead oil were equal.

In the absence of gravity and ignoring the pressure dependencies of the densities we can express the phase velocities in terms of the fractional flow functions

$$
\begin{aligned}
& u_{w}=\frac{\lambda_{w}}{\lambda_{w}+\lambda_{o}+\lambda_{g}} u+\frac{\lambda_{o} \lambda_{w}}{\lambda_{w}+\lambda_{o}+\lambda_{g}} \frac{\partial p_{c}^{o w}}{\partial x}+\frac{\lambda_{g} \lambda_{w}}{\lambda_{w}+\lambda_{o}+\lambda_{g}} \frac{\partial p_{c}^{g w}}{\partial x} \\
& u_{o}=\frac{\lambda_{o}}{\lambda_{w}+\lambda_{o}+\lambda_{g}} u-\frac{\lambda_{o} \lambda_{w}}{\lambda_{w}+\lambda_{o}+\lambda_{g}} \frac{\partial p_{c}^{o w}}{\partial x}+\frac{\lambda_{g} \lambda_{o}}{\lambda_{w}+\lambda_{o}+\lambda_{g}} \frac{\partial p_{g}^{g o}}{\partial x} \\
& u_{g}=\frac{\lambda_{g}}{\lambda_{w}+\lambda_{o}+\lambda_{g}} u-\frac{\lambda_{g} \lambda_{w}}{\lambda_{w}+\lambda_{o}+\lambda_{g}} \frac{\partial p_{c}^{g w}}{\partial x}-\frac{\lambda_{g} \lambda_{o}}{\lambda_{w}+\lambda_{o}+\lambda_{g}} \frac{\partial p_{g}^{g o}}{\partial x}
\end{aligned}
$$

Here $\lambda_{\alpha}$, with $\alpha=o, w, g$ are the phase mobilities i.e. the phase permeabilities divided by the viscosity and $p_{c}^{\alpha \beta}$ are the capillary pressures between the phases $\alpha$ and $\beta$. The capillary pressures are given as functions of the phase saturations (see e.g. [1]). Implementation of the capillary pressure term and the diffusion terms into the numerical schemes is straightforward, but in order to see its effects we need to implement a higher order scheme for the convection terms. This was not accomplished in due time and therefore we we will ignore these diffusional terms all together in our numerical schemes discussed below.

We need also to supplement a heat balance equation to obtain the temperature distribution. In our case the temperature distribution is measured and hence input in the mass balance equations. We also assume that pressure variations in the tube are small with respect to the applied pressure. Therefore the pressure $p^{a v}$ at which the process is carried out is also as considered given. We assume local thermodynamic equilibrium. According to Gibbs' phase rule there are two degrees of freedom i.e. $f=c-p+2=2$, because there are 
three components $c=3$ and three phases $p=3$. Therefore the densities are given functions of the temperature. These functions are summarized in the appendix.

4.2. Equations in the warm two phase zone. The mass balance equations for the three components in the two phase zone (aqueous and oleic) can be written as Equations 4.1,4.2 and 4.3 , but with $S_{g}=u_{g}=u_{g w}=u_{g v}=0$. Application of Gibbs' phase rule now leads to $f=c-p+2=3$, because there are three components and two phases. The pressure is given as the pressure at which the process is carried out and the temperatures are measured. Therefore we need to choose one independent thermodynamic variable e.g the composition of the liquid oil mixture.

4.3. Initial and boundary conditions. The equations are solved subject to the following conditions

\begin{tabular}{||l|l|l|l||}
\hline variable & $t=0$ & $x=0$ & $x=L$ \\
\hline$S_{w}$ & $S_{w}=S_{w c}$ & $u_{w}=0$ & \\
$S_{g}$ & $S_{g}=0$ & $u_{g}=0$ & \\
$T$ & $T=T^{o}$ & $T(x \rightarrow-\infty)=0$ & $T=T^{b}$ \\
$u=u_{w}+u_{o}+u_{g}$ & $u=0$ & $u=0$ & \\
\hline
\end{tabular}

5. MOdel FOR THE TEMPERATURE IN THE TUBE

We like to find a simple expression for the time and space dependent temperature within the tube. On the other hand we like to avoid the spurious effects inherent to a polynomial fit. Therefore we introduce a very simple heat conduction model of coupled heat flow in the central porous medium and the outside iron tube. Because we consider linear heat transfer between the porous medium (1) and the iron tube (2) we obtain a linear equation, for which we can easily derive the Laplace transformed temperature. We use the Stehfest algorithm for the inversion. The equations in dimensionless form read

$$
\begin{gathered}
\frac{\partial T_{1}}{\partial t}=\frac{\partial^{2} T_{1}}{\partial x^{2}}+M\left(T_{2}-T_{1}\right) \\
\chi \frac{\partial T_{2}}{\partial t}=\frac{\partial^{2} T_{2}}{\partial x^{2}}-N\left(T_{2}-T_{1}\right)
\end{gathered}
$$

where we use

$$
\begin{gathered}
\frac{T_{i}-T_{o}}{T_{b}-T_{o}}>T_{i}, \frac{\kappa_{1} t}{(\rho c)_{1} L^{2}}->t, \frac{x}{L}->x \\
\chi=\frac{(\rho c)_{2}}{(\rho c)_{1}} \frac{\kappa_{1}}{\kappa_{2}}, M=\frac{\beta}{\kappa_{1}} \frac{\left(T_{2}-T_{1}\right)}{\pi R_{1}^{2}}, N=\frac{\beta}{\kappa_{2}} \frac{\left(T_{2}-T_{1}\right)}{\pi\left(R_{2}^{2}-R_{1}^{2}\right)},
\end{gathered}
$$

We use as initial condition that $T=0$, and the boundary conditions that at $T_{i} \rightarrow 0$ at $x \rightarrow \infty$ and $T(x=1, t)=1$. Laplace transformation and the standard approach to solve coupled systems of linear ordinary differential equations leads to

$$
\begin{aligned}
& \widehat{T}_{1}=A(s) \exp \left(-\sqrt{y_{1}} z\right)+\left(\frac{1}{s}-A(s)\right) \exp \left(-\sqrt{y_{2}} z\right) \\
& \widehat{T}_{2}=C(s) \exp \left(-\sqrt{y_{1}} z\right)+\left(\frac{1}{s}-C(s)\right) \exp \left(-\sqrt{y_{2}} z\right)
\end{aligned}
$$




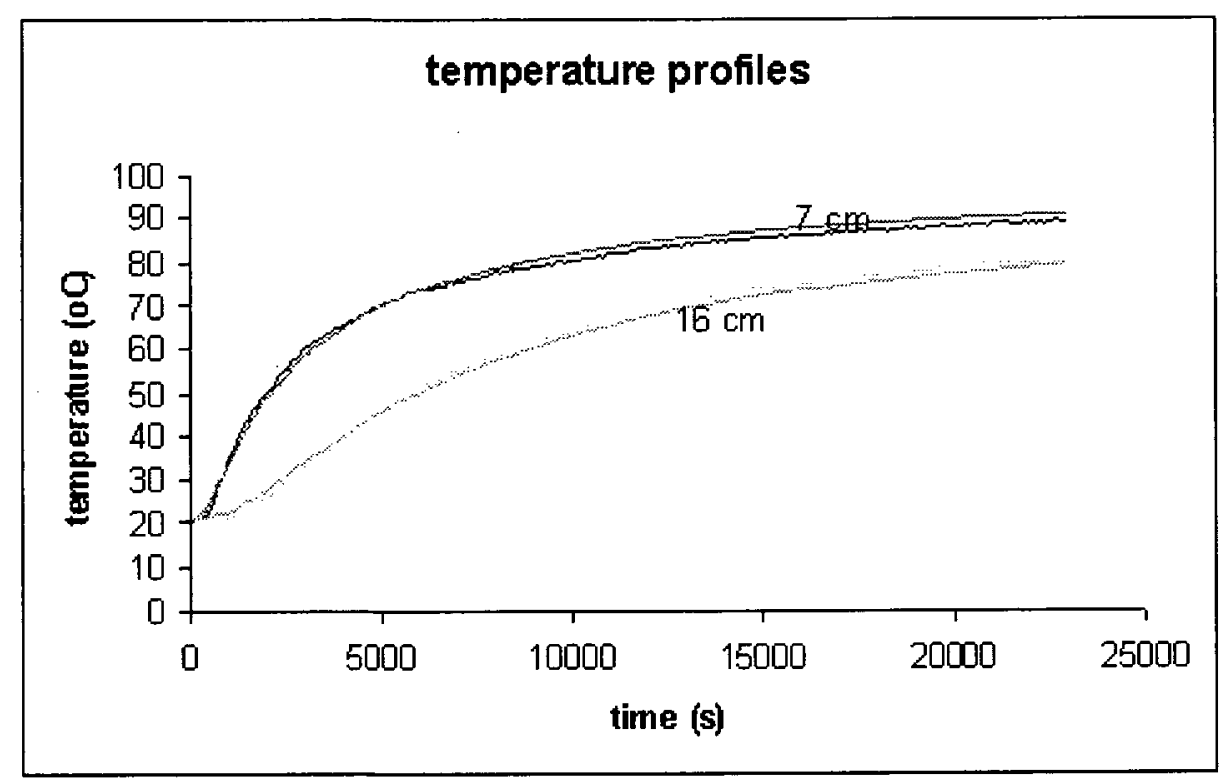

Figure 5.1. Comparison of experimental and model temperature profiles

with

$$
y_{1,2}=\frac{1}{2}((1+\chi) s+N+M) \pm \frac{1}{2} \sqrt{\left(((1+\chi) s+N+M)^{2}-4 \chi s^{2}-4 M \chi s-4 N s\right)}
$$

Resubstituting Equations 5.3 into the Laplace transformed version of Eqs. 5.1 and 5.2 leads to two equations with the unknowns $A(s)$ and $C(s)$. These expressions can be substituted into Eqs. 5.3 to obtain the Laplace transformed expressions for $\widehat{T}_{1}$ and $\widehat{T}_{2}$. We use the Stehfest algorithm to invert the equation from $\widehat{T}_{i}$ to $T_{i}$ with $i=1,2$. We use the temperature model that uses Laplace transforms for a optimal set of parameters. For this set of parameters there is good agreement between the experimental temperature profile and the computed temperature profile. The comparison between experimental and matched profiles is shown in Figure 5.1. We have used $(\rho c)_{2}=3.55 E+06 \mathrm{~J} / \mathrm{m}^{3} / K,(\rho c)_{1}=2.00 E 6 \mathrm{~J} / \mathrm{m}^{3} / K, \kappa_{1}=$ $2 W / m / K, \kappa_{2}=80.3 W / m / K, R_{1}=0.02 m, R_{2}=0.023 m, \mu=0.3$ and $L=0.3 m$.

\section{Numerical SOLUTION}

The mass balance equations and energy equation can be written in the general form

$$
\frac{\partial G_{c}}{\partial t}+\frac{\partial u F_{c}}{\partial x}=0 \text { with } c=w, v, d
$$

We use an equidistant grid with grid cell length $\Delta x$. The average values of the dependent variables attributed to the value at the center of the grid cell. The grid cells are numbered $i=1, N$, where the grid cell 1 is bounded at the left by a no flow barrier and the grid cell $N$ at the right is adjacent to the high permeable layer. We use an explicit scheme with upstream weighting i.e. the flux carries the properties of the center of the grid cell where 
the flow is coming from. Because in this case all flow is in the direction of grid cell $N$, we find the following numerical model

$$
G_{c}^{i, k+1}=G_{c}^{i, k}+\frac{u^{i-1, k+1} F_{c}^{i-1, k}-u^{i, k+1} F_{c}^{i, k}}{\Delta x}
$$

where $i$ is the center of the grid cell $i$ and $k$ denotes the time level. We note that we use the total Darcy velocity at the time level $k+1$. We start the procedure at grid cell 1 and use that the flow entering this cell, which may be denoted as $u^{0, k+1} F_{c}^{0, k}$, is zero. In the solution procedure we distinguish between the hot three phase zone and the warm two phase zone.

6.1. Hot three phase zone. In the hot three phase zone we use the measured temperature profile and subsequently compute all the densities at the given pressure and temperature. Subsequently we solve Equation 6.1 for the mass balance equations.

$$
\begin{gathered}
\varphi\left(\rho^{W} S_{w}+\rho^{g w} S_{g}\right)^{i, k+1}=\varphi\left(\rho^{W} S_{w}+\rho^{g w} S_{g}\right)^{i, k}+ \\
\left(u^{i-1, k+1}\left(\rho^{W} f_{w}+\rho^{g w} f_{g}\right)^{i-1, k}-u^{i, k+1}\left(\rho^{W} f_{w}+\rho^{g w} f_{g}\right)^{i, k}\right) \frac{\Delta t}{\Delta x}=0 \\
\varphi\left(\rho^{o v} S_{o}+\rho^{g v} S_{g}\right)^{i, k+1}=\varphi\left(\rho^{o v} S_{o}+\rho^{g v} S_{g}\right)^{i, k}+ \\
+\left(u^{i-1, k+1}\left(\rho^{o v} f_{o}+\rho^{g v} f_{g}\right)^{i-1, k}-u^{i, k+1}\left(\rho^{o v} f_{o}+\rho^{g v} f_{g}\right)^{i, k}\right) \frac{\Delta t}{\Delta x}=0 \\
\varphi\left(\rho^{o d} S_{o}\right)^{i, k+1}=\varphi\left(\rho^{o d} S_{o}\right)^{i, k}+\left(u^{i-1, k+1}\left(\rho^{o d} f_{o}\right)^{i-1, k}-u^{i, k+1}\left(\rho^{o d} f_{o}\right)^{i, k}\right) \frac{\Delta t}{\Delta x}=0
\end{gathered}
$$

Because the numerical mass balance equation 6.1 is linear in terms of $S_{w}^{i, k+1}, S_{g}^{k+1}$ and $u^{i, k+1}$ and the velocity $u^{i-1, k+1}$ is known when we applied our numerical scheme at the grid cell $i-1$, we can find explicitly the values of $S_{w}^{i, k+1}, S_{g}^{i, k+1}$ and $u^{i, k+1}$. It is possible that all water evaporates and that we have a two-phase three component hot zone. There will be an additional degree of freedom. On inspection of the above equations in particular equation 6.4 we observe that the equations are no longer linear in $\left(\rho^{o d}\right)^{i, k+1} S_{g}^{k+1}$ and $u^{i, k+1}$. Because of this complication we performed simulations until all the water was evaporated in the cell nearest to the outlet.

Remark 6.1. At the moment of transition in a grid cell from the two phase behavior to three phase behavior it appears as if the gas saturation changes with time to the power of an exponent smaller than one i.e. the derivative versus time is infinite. Hence there is a very large increase in the gas saturation, and this time step can lead to somewhat inaccurate results. This aspect deserves further investigation.

Remark 6.2. When the cell just upstream of a three phase cell is converted from a twophase status to a three phase status there appears to be a small but sudden decrease of the gas saturation. This aspect also requires further attention.

Remark 6.3. When all water has evaporated we need a different scheme, which has not been elaborated here 
6.2. Warm two phase zone. The numerical model of the water balance equation in the warm two phase zone reads

$$
\varphi S_{w}^{i, k+1}=\frac{\varphi\left(\rho^{W} S_{w}\right)^{i, k}+\left(u^{i-1, k+1}\left(\rho^{W} f_{w}\right)^{i-1, k}-u^{i, k+1}\left(\rho^{W} f_{w}\right)^{i, k}\right) \frac{\Delta t}{\Delta x}}{\rho^{i, k+1, W}}
$$

We write a similar equation for the volatile oil balance equation, but instead we divide by $\rho^{i, k+1, o V}$ i.e. the density of pure volatile oil in the oleic phase. We do the same for the dead oil, but now we divide by $\rho^{i, k+1, o D}$ i.e. the density of pure dead oil in the oleic phase. Subsequently we add the equations and we obtain, using the mixing rule equation 3.1

$$
\begin{gathered}
\varphi S_{o}^{i, k+1}\left(\frac{\rho^{i, k+1, o v}}{\rho^{i, k+1, o V}}+\frac{\rho^{i, k+1, o d}}{\rho^{i, k+1, o D}}\right)=\varphi S_{o}^{i, k+1}= \\
\frac{\varphi\left(\rho^{o v} S_{o}\right)^{i, k}+\left(u^{i-1, k+1}\left(\rho^{o v} f_{o}\right)^{i-1, k}-u^{i, k+1}\left(\rho^{o v} f_{o}\right)^{i, k}\right) \frac{\Delta t}{\Delta x}}{\rho^{i, k+1, o V}} \\
\frac{\varphi\left(\rho^{o d} S_{o}\right)^{i, k}+\left(u^{i-1, k+1}\left(\rho^{o d} f_{o}\right)^{i-1, k}-u^{i, k+1}\left(\rho^{o d} f_{o}\right)^{i, k}\right) \frac{\Delta t}{\Delta x}}{\rho^{i, k+1, o D}}
\end{gathered}
$$

We note that $S_{o}^{i, k+1}+S_{w}^{i, k+1}=1$. Given the temperature we note that the only two unknowns left are $S_{w}^{i, k+1}$ and $u^{i, k+1}$, also because $u^{i-1, k+1}$ was already evaluated when we dealt with cell $i-1$. The linear equation in terms of the two unknowns can be solved easily. The composition can be found from either the numerical equation for the volatile oil or for the dead oil. If the temperature starts to exceed the temperature at which the volatile oil only exists in the liquid phase we need to go back and solve the equations for the three phase hot temperature case.

\section{REsults}

We used the numerical method described above to obtain the saturation curves after 1.04 days of heating. The curve indicated with $z$ shows the volume fraction of volatile oil in the oil mixture. In the simulation we use 25 grid cells. The result is shown in Figure 7.1. The tube is heated at a distance of $0.3 \mathrm{~m}$ from the closed end of the tube. The gas saturation at small distances shows the two effects mentioned above. First we observe that the gas saturation rises steeply after the temperature at which three phases can coexist has been reached. The small dip at 0.05 meter shows the effect of the cell upstream being converted from the two phase to the three phase condition. As the water saturation approaches zero at a distance of about $0.25 \mathrm{~m}$ leads to other a sudden change in direction of the curve. This is most clearly observed in water saturation curve. As all cells are only influenced by the cells upstream, we assert that the rest of the profile gives a reasonable representation of the saturation profiles. The oil saturation remains relatively constant but the composition changes from thirty percent at the closed end to almost zero at the open end. Therefore the dead oil shows a minimum in the middle of the tube. There is some production of dead oil, but the larger part remains behind. Further work is necessary to see whether this is more favorable when much lower volume fractions of dead oil are present 


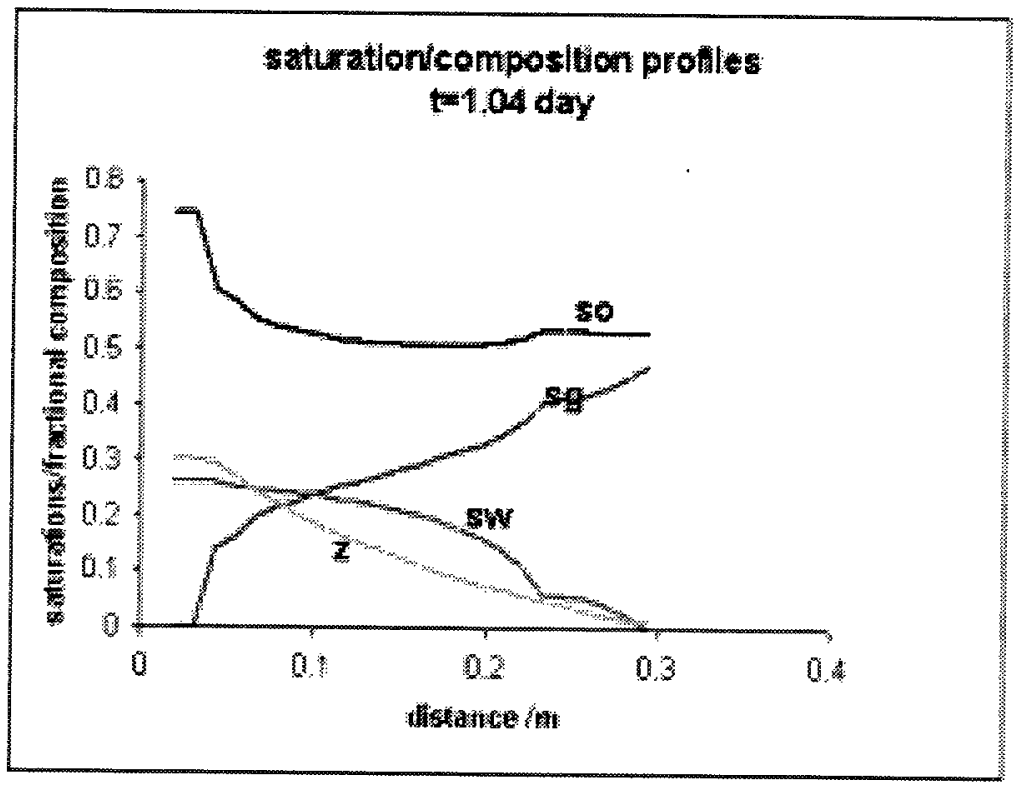

FIGURE 7.1. Saturation and composition profiles obtained from the numerical model

\section{CONCLUSIONS}

(1) A numerical model for the interpretation of laboratory experiments designed to quantify oil recovered from low permeable layers or lenses has been developed.

(2) Large parts of dead oil remain unrecovered at least for the high initial volume fraction of dead oil, which was taken as an example. The total range of volume fractions must be studied for a full quantification

(3) The numerical model shows some features that deserve further study. These features are related to the conversion from two-phase to three phase behavior and the full evaporation of water.

(4) Combination of experimental laboratory work and modelling provides insight in the complex mechanisms involved in thermally enhanced multi-phase processes.

\section{Physical quantities; symbols and values}

In the Tables we summarize the values and units of the various quantities used in the computation and empirical expressions for the various parameter functions. 


\begin{tabular}{|c|c|c|c|}
\hline Physical quantity & Symbol & Value & $\overline{\text { Unit }}$ \\
\hline Fractional flow water & $f_{w}$ & $\frac{k_{r w}}{\mu^{W}} /\left(\frac{k_{r w}}{\mu^{W}}+\frac{k_{r o}}{\mu^{\text {oll }}}+\frac{k_{r g}}{\mu_{g}}\right)$ & {$[-]$} \\
\hline Fractional flow gas & $f_{g}$ & $\frac{k_{r g}}{\mu_{g}} /\left(\frac{k_{r w}}{\mu^{W}}+\frac{k_{r o}}{\mu^{\text {oil }}}+\frac{k_{r g}}{\mu_{g}}\right)$. & {$[-]$} \\
\hline Porous rock permeability & $k$ & $1.0 \times 10^{-12}$ & {$\left[\mathrm{~m}^{3}\right]$} \\
\hline Atmospheric Pressure & $p^{b}$ & $1.0135 \times 10^{5}$ & {$[\mathrm{~Pa}]$} \\
\hline Water, steam phase velocity & $u_{w}, u_{g}$ & $u f_{w}, u f_{g}$ & {$\left[\mathrm{~m}^{3} /\left(\mathrm{m}^{2} \mathrm{~s}\right)\right]$} \\
\hline velocity & $u$ & $u_{w}+u_{g}+u_{o}$ & {$\left[\mathrm{~m}^{3} /\left(\mathrm{m}^{2} \mathrm{~s}\right)\right]$} \\
\hline Porosity & $\varphi$ & 0.35 & {$[-]$} \\
\hline oir temperature & $T^{0}$ & 293. & {$[\mathrm{~K}]$} \\
\hline Boiling points water/vol oil & $T^{b W}, T^{b V}$ & $373.15,371.57$. & {$[\mathrm{K}]$} \\
\hline Gas constant & $R$ & 8.31. & {$[\mathrm{~J} / \mathrm{mole} / \mathrm{K}]$} \\
\hline Liquid vol. oil mole fraction & $x^{o v}$ & $\frac{\rho^{o v} / M^{V}}{\rho^{o v} / M^{V}+\rho^{o d} / M^{D}}$ & {$[-]$} \\
\hline conductivity & $\kappa_{r}$ & 2.0 & {$[\mathrm{~W} /(\mathrm{mK})]$} \\
\hline $\mathrm{Wa}$ & $\mu^{W}$ & $\ln \mu^{W}=-12.06+\frac{1509}{T}$. & {$[\mathrm{Pa} \mathrm{s}]$} \\
\hline cosity & $\mu^{o V}$ & $\ln \mu^{o V}=-10.813+\frac{880.2}{T}$. & {$[\mathrm{Pa} \mathrm{s}]$} \\
\hline Dead oi & $\mu^{o D}$ & $\ln \mu^{o D}=-13.79+\frac{3781}{T}$ & {$[\mathrm{~Pa} \mathrm{~s}]$} \\
\hline Gas & $\mu_{g}$ & $3.2 \times 10^{-5}\left(\frac{T}{T^{b}}\right)^{0.6}$ & {$[\mathrm{~Pa} \mathrm{~s}]$} \\
\hline Wate & $\rho^{W}$ & $998.2 \exp \left(-\alpha\left(T-T^{o}\right)\right)$ & {$\left[\mathrm{kg} / \mathrm{m}^{3}\right]$} \\
\hline density & $\rho^{o V}$ & $683 \cdot \exp \left(-\alpha\left(T-T^{o}\right)\right)$ & {$\left[\mathrm{kg} / \mathrm{m}^{3}\right]$} \\
\hline Dead oil density & $\rho^{o D}$ & $800 \cdot \exp \left(-\alpha\left(T-T^{o}\right)\right)$ & {$\left[\mathrm{kg} / \mathrm{m}^{3}\right]$} \\
\hline steam concentration & $\rho^{g w}$ & $M^{W} P^{g w} /\left(R T_{z}\right)$ & {$\left[\mathrm{kg} / \mathrm{m}^{3}\right]$} \\
\hline oil vapor concentration & $\rho^{g v}$ & $\rho^{g v}=\rho^{g V}\left(1-\frac{\rho^{g w}}{\rho^{g W}}\right)$ & {$\left[\mathrm{kg} / \mathrm{m}^{3}\right]$} \\
\hline Volatile oil density in hot zone & $\rho^{o v}(T)$ & $x^{o v}$ and Eq. (3.2) & {$\left[\mathrm{kg} / \mathrm{m}^{3}\right]$} \\
\hline Dead oil density in hot zone & $\rho^{o d}$ & $\rho^{o d}=\rho^{o D}\left(1-\frac{\rho^{o v}}{\rho^{o V}}\right)$ & {$\left[\mathrm{kg} / \mathrm{m}^{3}\right]$} \\
\hline steam density & $\rho^{g W}$ & $M^{W} P^{t o t} /\left(R T_{z}\right)$ & {$\left[\mathrm{kg} / \mathrm{m}^{3}\right]$} \\
\hline Isity & $\rho^{g V}$ & $M^{V} P^{t o t} /\left(R T_{z}\right)$ & {$\left[\mathrm{kg} / \mathrm{m}^{3}\right]$} \\
\hline$n$ heat vol. oil & $\Lambda^{V}(T) / 10^{5}$ & $(3.648-0.00584(T-298))$ & {$[\mathrm{J} / \mathrm{kg}]$} \\
\hline Evaporation heat water & $\Lambda^{W}(T) / 10^{6}$ & $(2.444-0.00222(T-298))$ & {$[\mathrm{J} / \mathrm{kg}]$} \\
\hline Molecular Wts & $M^{W}, M^{D}, M^{V}$ & $0.018,0.4,0.10021$ & {$[\mathrm{~kg} / \mathrm{mole}]$} \\
\hline Liquid oil viscosity & $\mu^{o i l}$ & $\left(\zeta^{o v}\left(\mu^{o V}\right)^{\frac{1}{4}}+\zeta^{o d}\left(\mu^{o D}\right)^{\frac{1}{4}}\right)^{4}$ & {$[\mathrm{~Pa} \mathrm{~s}]$} \\
\hline Volume fractions & $\zeta^{g v}, \zeta^{g w}, \zeta^{o v}, \zeta^{o d}$ & $\frac{\rho^{g v}}{\rho^{g V}}, \frac{\rho^{g w}}{\rho^{g W}}, \frac{\rho^{o v}}{\rho^{o V}}, \frac{\rho^{o d}}{\rho^{o D}}$ & {$[-]$} \\
\hline Azec & $T_{z}$ & Eq. (3.2). & {$[\mathrm{K}]$} \\
\hline Liquid Thermal expansion & $\alpha$ & $5 \times 10^{-4}$ & {$[/ \mathrm{K}]$} \\
\hline Gas Thermal expansion. & $\alpha^{g v}, \alpha^{g w}$ & $\left(\frac{-1}{\rho^{g c}}\right)\left(\frac{\partial \rho^{g c}}{\partial T}\right)_{p}$ & {$[/ \mathrm{K}]$} \\
\hline Pure Gas Thermal expansion. & $\alpha^{g V}, \alpha^{g W}$ & $\left(\frac{-1}{\rho^{g C}}\right)\left(\frac{\partial \rho^{g C}}{\partial T}\right)_{p}=\frac{1}{T}$. & {$[/ \mathrm{K}]$} \\
\hline Enthalpy vol. oil/dead oil & $h^{o V}, h^{o D}$ & $2242\left(T-T^{o}\right), 2000\left(T-T^{o}\right)$ & {$[\mathrm{J} / \mathrm{kg}]$} \\
\hline Enthalpy & & $4184\left(T-T^{o}\right)$ & {$[\mathrm{J} / \mathrm{kg}]$} \\
\hline Enthalpy steam/oil vapor & $h^{g W}, h^{g V}$ & $\begin{array}{l}1966\left(T-T^{o}\right), 1657\left(T-T^{o}\right) \\
30 \times 10^{6}\end{array}$ & {$[\mathrm{~J} / \mathrm{kg}]$} \\
\hline $\begin{array}{l}\text { sorting factor } \\
\text { Rock enthalpy }\end{array}$ & $\begin{array}{l}\lambda_{s} \\
(\rho h)^{R}\end{array}$ & $\begin{array}{l}3.0 \times 10^{0} \\
2.029 \times 10^{6}\left(T-T^{o}\right)\end{array}$ & $\begin{array}{r}{\left[\mathrm{J} /\left(\mathrm{m}^{-} \mathbf{h}\right)\right]} \\
{\left[\mathrm{J} /\left(\mathrm{m}^{3}\right)\right]}\end{array}$ \\
\hline
\end{tabular}

The three phase relative permeabilities are obtained by combining Corey two phase relative permeabilities and the modified Stone I method, see Fayers \& Matthews [6]. In full dimensional form they read: 
Here

$$
\begin{aligned}
& k_{\mathrm{rw}}=k_{\mathrm{rw}}\left(S_{\mathrm{w}}\right)=k_{\mathrm{rw}}^{\prime} S_{\mathrm{we}}^{\frac{2+3 \lambda_{s}}{\lambda_{s}}} \\
& k_{\mathrm{rg}}=k_{\mathrm{rg}}\left(S_{\mathrm{g}}\right)=\quad k_{\mathrm{rg}}^{\prime} S_{\mathrm{ge}}^{2}\left(1-\left(1-\dot{S}_{\mathrm{ge}}\right)^{\frac{2+\lambda_{s}}{\lambda_{s}}}\right) \\
& k_{\mathrm{ro}}=k_{\mathrm{ro}}\left(S_{\mathrm{w}}, S_{\mathrm{g}}\right)=\frac{S_{\mathrm{o}}}{k_{\mathrm{rcow}}\left(1-S_{\mathrm{w}}\right)\left(1-S_{\mathrm{ge}}\right)} k_{\mathrm{row}} k_{\mathrm{rog}}
\end{aligned}
$$

and

$$
S_{\mathrm{we}}=\frac{S_{\mathrm{w}}-S_{\mathrm{wc}}}{1-S_{\mathrm{wc}}}, S_{\mathrm{ge}}=\frac{S_{\mathrm{g}}}{1-S_{\mathrm{wc}}}
$$

$$
k_{\mathrm{row}}=k_{\mathrm{rg}}^{\prime}\left(1-S_{\mathrm{we}}\right)^{2}\left(1-S_{\mathrm{we}}^{\frac{2+\lambda_{s}}{\lambda_{s}}}\right), k_{\mathrm{rog}}=k_{\mathrm{rw}}^{\prime}\left(1-S_{\mathrm{ge}}\right)^{\frac{2+3 \lambda_{s}}{\lambda_{s}}} .
$$

We use $k_{\mathrm{rw}}^{\prime}=0.5$ for the end-point permeability of the wetting phase at residual non-wetting phase saturation and $k_{\mathrm{rg}}^{\prime}=1.0$ for the end-point permeability of the non-wetting phase at connate wetting phase saturation. Finally we set $k_{\text {rcow }}=1$. To describe the effect of oil film flow (oil may spread on water in the presence of steam), the expressions for $k_{\text {row }}$ and $k_{\text {rog }}$ are different from the ones proposed by Fayers and Matthews [6].

\section{REFERENCES}

[1] BEAR, J., Dynamics of Fluids in Porous Media, Dover Publications, Inc., New York (1972).

[2] BIRD, R.B., STEWART, W.E., and LIGHTFOot, E.N., Transport Phenomena, John Wiley \& Sons (1960).

[3] BROOKS, R.H., and COREY, A.T., Properties of porous media affecting fluid flow, J. Irr. Drain. Div., Proc., Am. Soc. Civ. Eng. June (1966), 61-88.

[4] BRUINING, J., and DUIJN, C.J., Uniqueness Conditions in a Hyperbolic Model for Oil Recovery by Steamdrive, Computational Geosciences 4, 65-98 (2000).

[5] BRUINING, J., and MARCHESIN, D., Nitrogen-steam injection in a porous medium with water, Publication in preparation (2002).

[6] Fayers, F.J. and Matthews, J.D., Evaluation of Normalized Stone's Methods for Estimating Three-Phase Relative Permeabilities, Soc. Pet. Eng. J., (1984) 224-232.

[7] GodderiJ, R.R.G.G., A Three Dimensional Interface Model for Steamdrive in Heterogeneous Reservoirs, (1997), Ph-D thesis of Delft Univeristy of Technology.

[8] GODDERIJ, R.R.G.G., BRUining, J., and molenaAR, J., A Fast $3 D$ Interface Simulator for Steamdrives, SPE Journal bf 4 (4), December (1999)

[9] GODDERIJ, R.R.G.G., GUMRAH, F., PALMGREN, C.T.S., BRUINING, J., An investigation of the vertical sweep efficiency of steam drive in a layered reservoir, In: H.J. de Haan (ed.), New developments in improved oil recovery, ISBN 1-897799-22-5, The Geological Society, London, UK, p. 261-273 (1995).

[10] MANDL, G.W., and VOLEK, C.W., Heat and mass transport in steamdrive processes, SPEJ, 57-79, March 1969.

[11] PALmgREn, C.T.S., Oil Recovery by Steamdrive; An interface approach, (1992), Ph-D thesis of Delft Univeristy of Technology

[12] TORTIKE, W.S., and FAROUQ ALI, S.M., Saturated-steam-property functional correlations for fully implicit reservoir simulation, SPERE 4(4) (November 1989) 471-474.

[13] WILlmaN, B.T.,VAlleroy, V.V., RUNBERG, G.W., CORNEliUs, A.J., and POWERS, L.W., Laboratory Studies of Oil Recovery by Steam Injection, J. Pet. Tech. (July 1961) $681-690$ 
